Cahiers « Mondes anciens »

ANCIENS

Histoire et anthropologie des mondes anciens

15 | 2022

Les parures divines

\title{
Chic ou toc? La fonction des bijoux déposés dans les sanctuaires grecs
}

Chic or Fake Jewels? The Function of Jewellery in Greek Sanctuaries

\section{Clarisse Prêtre}

\section{(2) OpenEdition}

1 Journals

Édition électronique

URL : https://journals.openedition.org/mondesanciens/3732

DOI : 10.4000/mondesanciens.3732

ISSN : 2107-0199

Éditeur

UMR 8210 Anthropologie et Histoire des Mondes Antiques

\section{Référence électronique}

Clarisse Prêtre, "Chic ou toc ? La fonction des bijoux déposés dans les sanctuaires grecs », Cahiers «Mondes anciens » [En ligne], 15 | 2022, mis en ligne le 18 janvier 2022, consulté le 21 janvier 2022. URL : http://journals.openedition.org/mondesanciens/3732 ; DOI : https://doi.org/10.4000/ mondesanciens.3732

Ce document a été généré automatiquement le 21 janvier 2022.

Les Cahiers «Mondes Anciens » sont mis à disposition selon les termes de la licence Creative Commons Attribution - Pas d'Utilisation Commerciale - Pas de Modification 4.0 International. 


\title{
Chic ou toc? La fonction des bijoux déposés dans les sanctuaires grecs
}

Chic or Fake Jewels? The Function of Jewellery in Greek Sanctuaries

\author{
Clarisse Prêtre
}

\section{Introduction}

1 L'étude des objets dédiés dans les sanctuaires grecs met souvent en exergue une relation bijective entre le donateur et la divinité bénéficiaire, selon le principe maussien déjà ancien d'un système d'échanges contraints autour de dons et de contredons ${ }^{1}$. D'un autre côté, on théorise sur le lien nécessaire entre l'offrande et son auteur, en spéculant - à tort - sur une répartition genrée, qui, lorsqu'elle est poussée à son paroxysme, fait supposer que certains dons sont typiquement masculins ou typiquement féminins, sans prendre en compte le caractère polymorphique de l'offrande.

2 Si les pans donateur/divinité et donateur/offrande ont fait l'objet de multiples théories (Prêtre 2009), le dernier axe qui lie l'offrande à la divinité demeure abordé de façon moins systématique: la confrontation matérialité/immatériel semble trouver ses limites en l'absence du medium donateur, seul apte à laisser des traces de ses motivations personnelles.

3 Certes, certains courants de pensée ont aussi appliqué l'argument genré à l'autre extrémité de la chaîne votive dans un raisonnement unilatéral cette fois-ci : une arme sera destinée à un dieu, un bijou à une déesse ${ }^{2}$. De fait, les sources textuelles et archéologiques valident en apparence cette triple connexion entre la femme, la déesse et la parure, pour ne citer que l'exemple qui nous intéresse ici. Entre la fin de l'enfance, le mariage, l'enfantement, l'envergure des préoccupations qui gravitent autour de la sphère féminine explique la dédicace d'objets personnels tels que les miroirs, les rubans, les bijoux ou les vêtements à des divinités aptes à protéger les femmes et à les assister lors des multiples étapes de leur vie ${ }^{3}$. Ce schéma mental trouve sa source dans les textes antiques évoquant ce principe d'association genrée : ainsi dans l'hymne Sur 
les bains de Pallas, Callimaque souligne le problème du caractère prédéterminé des offrandes en fonction de la divinité : les jeunes filles argiennes qui baignent la statue d'Athéna peuvent l'oindre d'huiles mais son profil martial interdit qu'on lui offre des mélanges sophistiqués: son corps athlétique semblable à celui d'un jeune homme réclame davantage de l'huile d'olive pure, à l'instar de celle qu'utilisent Castor et Héraclès. De la même façon, tout ce qui pourrait exacerber une beauté artificielle, la féminité et la sexualité est exclu du rite argien, Callimaque rappelant que le miroir est l'attribut d'Aphrodite (Sur les bains de Pallas, v. 15-17).

4 Dans la réalité antique cependant, l'interchangeabilité des offrandes entre les différentes divinités n'est plus à démontrer et dépasse le clivage moderne masculin/ féminin que certains ont tenté - à tort - d'appliquer aux différents acteurs de l'acte dédicatoire tout comme aux divinités: Héra peut recevoir les mêmes offrandes de bijoux qu'Artémis (Grubbs et Parkin 2013, p. 180-181), un homme peut dédier à Artémis sa couronne de vainqueur aux concours (IG V, 1293$)$; le spectre des variations dans le «triangle votif», pour reprendre l'expression de François de Polignac (2009), est de grande envergure.

5 Au-delà du caractère labile de la relation tissée entre le donateur et la divinité, l'anathem $a^{4}$ constitue donc le cœur de l'acte dédicatoire et permet une grille de lecture polyphonique du système votif. Qu'il soit élaboré à des fins strictement religieuses ou issu du détournement d'un objet ordinaire reconverti pour être dédié, le don matériel a pour fonction première de plaire à la divinité récipiendaire, dans une démarche propitiatoire ou gratulatoire ${ }^{5}$. Il devient alors la propriété du dieu et revêt de facto un caractère sacré.

6 Néanmoins, selon des critères endogènes liés à l'identité du bénéficiaire comme à la nature de la dédicace, l'offrande déposée dans un temple peut parfois jouer un rôle complexe, à cheval entre l'utilitaire, le rituel et le sacré. Nous nous étions déjà posé la question de ses multiples fonctions (Prêtre 2014), sans nous attacher à un type d'offrande précis; il est nécessaire de voir dorénavant si une catégorie particulière d'objets dédiés confirme cette idée de rôles polyvalents. Cependant, il ne s'agira pas ici de s'intéresser aux destinations utilitaires de l'artefact en contexte rituel, mais plutôt de l'observer en lien avec le geste votif dans lequel il s'inscrit. Si on reprend l'énumération faite par Pausanias des offrandes déposées dans le temple de Zeus Olympien, force est de constater l'ambivalence de bon nombre d'entre elles: ainsi, le bouclier en or offert par les Lacédémoniens après leur victoire de Tanagra est au centre du fronton et l'inscription qui est sur le bouclier nous apprend par qui il a été dédié et à quelle occasion (Pausanias, V, 10,4); objet de gratitude après des faits d'armes, le bouclier est alors également un outil de propagande politique mais sa place au-dessus d'une statue de Nikè en or pose la question de sa véritable destination : s'agit-il d'une simple offrande commémorative à Zeus ou concerne-t-il plutôt Nikè, qu'il magnifie ? En d'autres termes, doit-il être assimilé aux quantités de boucliers votifs enregistrés dans les inventaires des temples attiques ${ }^{6}$ ou possède-t-il une supra-fonction en servant à la fois d'espace publicitaire, de dédicace officielle à Zeus et d'ex-voto subliminal destiné à plaire à Niké ? De façon plus complexe encore, les statues offertes brouillent la finalité de leur dédicace comme le résume Pausanias :

Me voici maintenant arrivé à la description des statues érigées à des particuliers, et de celles qui sont des offrandes aux dieux. Je n'ai pas cru devoir mêler ce que j'ai à en dire, comme je l'ai fait en décrivant la citadelle d'Athènes : dans cette dernière, en effet, les statues d'hommes sont comme tout le reste, des offrandes faites à la 
divinité, tandis que dans l'Altis, les statues érigées en l'honneur du dieu se distinguent des statues d'hommes qui sont, par elles-mêmes, une espèce de récompense accordée aux vainqueurs (Pausanias, V, 21, 1).

7 Le problème de la confusion moderne entre les statues offertes et les statues de culte a été pointé par Ioannis Mylonopoulos (2009) il y a quelques années lorsqu'il a recensé les différents moyens de distinguer dans un temple ce qui était la statue que l'on honorait (celle qu'on appelle communément la statue de culte en terminologie contemporaine) de ce qui pouvait être une statue offerte par le demos, par une fondation, une association voire un particulier mais qui n'était pas l'objet d'une vénération commune. Selon lui, une approche émique fondée sur la sémantique et la terminologie semblait difficile et il préconisait davantage une approche étique tenant compte d'une étude combinée de la position de la statue dans le temple, de son apparence et surtout de son «implication» dans les différents phénomènes cultuels et rituels. Évidemment cette méthodologie se heurte à des cas particuliers qui sortent du cadre d'analyse de Mylonopoulos, mais cela souligne bien que l'idée figée que nous avons aujourd'hui d'une statue de culte ne recouvre pas l'imaginaire antique, beaucoup plus fluide et mouvant en ce qui concernait la perception des images divines et leurs fonctions et usages.

8 Partant, dans une perspective complémentaire et non contradictoire de celle de Mylonopoulos, l'approche sémantique ne semble pas incompatible avec une réflexion sur les statues de culte. Il ne s'agira pas ici de s'intéresser aux termes qui les désignent elles-mêmes mais bien plutôt à la sphère physique dans laquelle elles gravitent: spatialité d'un temple, mise en scène du mobilier à l'intérieur de celui-là, instrumentum servant à les nettoyer chaque année, les champs sémantiques de la matérialité religieuse sont nombreux. Celui de la parure offre un terrain d'étude idéal, dans la mesure où les inventaires sacrés de la Grèce antique ont soigneusement enregistré les bijoux déposés dans les temples, en condition votive stricte ou pour également orner les statues de culte; il n'est plus à démontrer en effet que l'analyse terminologique des offrandes recensées dans les sanctuaires offre des clefs de compréhension pertinentes du système votif et cultuel.

9 Les inventaires de Délos constituent un creuset inépuisable pour étudier les parures à travers les descriptions qui en sont faites. Ainsi, à travers l'examen de certains aspects de la syntaxe votive des inventaires, il s'agira d'identifier les critères qui permettent de comprendre l'usage des bijoux après leur dédicace ; leur statut sacré étant définitif et invariable à partir du moment où ils sont offerts aux divinités, on essayera de voir si une remise en perspective et une analyse combinée de la matière, de la facture ou encore de la présentation dans les temples sont des éléments qui contribuent à appréhender la fonction des parures dédiées : offrandes par "destination », fabriquées à des fins votives et pour orner les statues des divinités honorées dans les temples, ou offrandes par " transformation », extraites du quotidien pour être dédiées et donc pour plaire aux divinités (Morel 1992, p. 221-232).

\section{Mise au point sémantique sur les termes kosmos/ kosmesis et kosmema}

10 Il existe un amalgame fréquent qui engendre des contresens dans l'interprétation de certains textes et par ricochet sur les actions et objets décrits par ces termes. 


\section{Kosmos, « parure », « collier ${ }^{7}$ »}

11 Sans refaire la genèse sémantique du mot kosmos, il est utile de rappeler que dès les sources homériques, quand il est employé en expression quasi adverbiale, il désigne le bon ordre, la bonne façon d'agencer, et pour reprendre les termes de Michel Casevitz (1989), « le kosmos est concrètement un (bon) arrangement, un agencement, une mise en ordre, qui par l'ordre même qui est ajouté à l'état naturel, devient parure, mise en beauté ». C'est également ainsi qu'on le comprend la plupart du temps dans les textes ultérieurs : c'est un des outils de séduction de la femme.

12 À la fois parure et comportement, il peut, entre autres, désigner l'ensemble des différentes pièces dont se pare une femme ou une déesse.

13 Chez Homère, le terme est employé lorsqu'Héra utilise les stratagèmes de séduction propres à Aphrodite :

Enfin, quand elle a ainsi autour de son corps disposé toute sa parure (кóøuov), elle sort de sa chambre, elle appelle Aphrodite, à l'écart des dieux (Iliade, XIV, v. 187-189).

Chez Euripide, le kosmos est lié aux vêtements revêtus par Alceste avant de mourir :

Quand elle a senti que le jour fatal était venu, d'une eau courante elle a baigné son beau corps, et tirant de coffres de cèdre vêtements et parure, s'en est parée avec décence (Alceste, 158-160).

Chez Démosthène le kosmos est évoqué à propos de la loi de Solon contre la femme adultère :

Solon, le plus illustre des législateurs, a traité, avec la gravité propre à ces temps

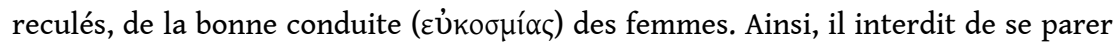

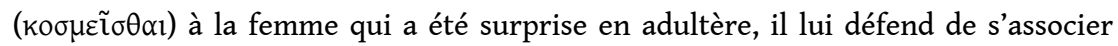
aux sacrifices publics, de peur qu'en se mêlant aux femmes honnêtes elle ne les corrompe. Si, en dépit de cette défense, elle prend part à ces cérémonies ou se pare

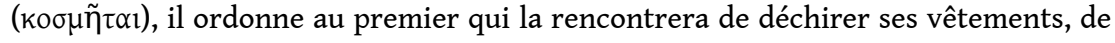

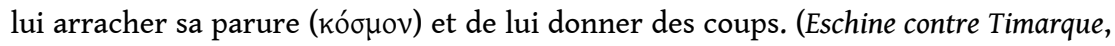
183).

Tous ces textes démontrent le caractère non naturel du kosmos. Le corps paré de différents ornements devient le marqueur d'une certaine condition, qu'elle soit humaine ou divine :

Du passage d'un corps nu à un corps paré (une parure quelle qu'elle soit), il y a l'artifice, une œuvre de mêtis, d'intelligence rusée, l'utilisation d'une technê. La kosmêtikê technê est ainsi précisément l'art de mettre en ordre, de s'arranger, de se parer ; il définit l'art de la toilette (Bodiou et al. 2011, p. 8).

Dans les sources épigraphiques, et notamment celles de Délos, il y a souvent amalgame entre le kosmos et la kosmesis, le premier constituant un ensemble d'objets destinés à parer une statue de culte; les seules occurrences qui ne concernent pas directement une divinité sont réservées à la description du collier dit « d'Ériphyle »"

18 - Dans l'inventaire de l'Artémision, entre la geranos ${ }^{9}$ et une épingle en argent: « la

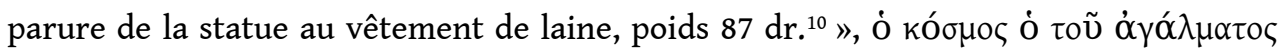

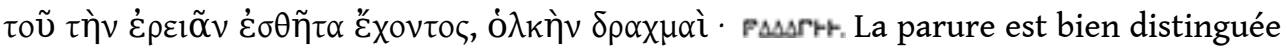
du vêtement porté par la statue, vraisemblablement, selon Philippe Bruneau (1970), « un xoanon de bois, seul genre d'idoles qu'on eût coutume d'habiller"11 ». 
19 - Dans l'inventaire de l'Artémision, entre différents éléments en or : «parure en or

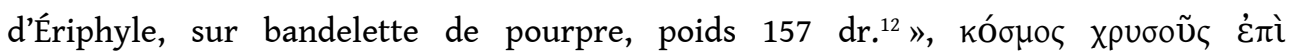

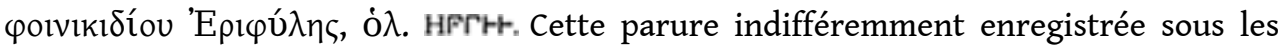

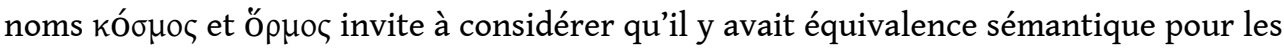
rédacteurs des inventaires entre les deux termes et donc, assurément, parenté stylistique et technique. Les autres occurrences du mot ópuoৎ renvoient à des colliers composites, tantôt agrémentés de pendeloques (amphores, motifs zoomorphes etc. comme la parure d'Eriphyle), tantôt avec des pierres serties en grand nombre.

20 Parmi les autres sources épigraphiques, un décret attique du $\mathrm{IV}^{\mathrm{e}} \mathrm{s}$. enregistre

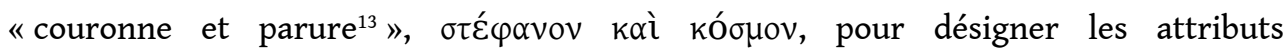
ornementaux d'une statue. Dans un autre décret trouvé au Pirée ${ }^{14}$, on déclare « que les porteuses de phiales et celles qui s'occupent de la déesse ajoutent une parure en argent

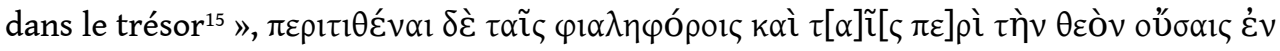

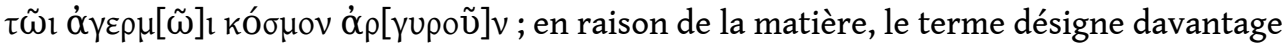
un seul bijou qu'un ensemble : en effet, dans le cas d'une parure composée, les éléments qui la constituent sont rarement tous du même métal et les catalogues sacrés ne manquent pas de détailler les incrustations et les sertissages divers.

21 En confrontant les témoignages littéraires aux sources iconographiques ${ }^{16}$, on constate

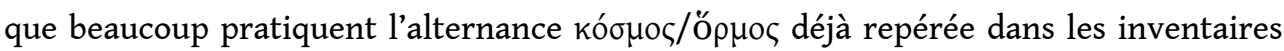
déliens pour nommer la parure d'Ériphyle : Athénée ne marque aucune différence entre l'un et l'autre (VI, 22, lignes 6 et 21) et Platon emploie le mot ǒ $\rho$ os pour évoquer la parure légendaire (République VII, 590). Le terme " parure » pour les inventaires déliens est donc trop approximatif et il faut plutôt comprendre qu'il s'agit d'un élément joaillier particulier - un collier - et non d'un bijou interchangeable. Ce collier n'est toutefois pas d'un type précis, qui l'aurait fait désigner par un terme spécifique dans les inventaires, mais un collier suffisamment exceptionnel, par sa composition ou son origine mythique, pour ne pas pouvoir appartenir à une simple donatrice.

\section{Kosmesis, « décoration »}

À côté des objets constituant la parure des statues et donc son kosmos, la kosmesis rassemble davantage une série de gestes et d'actions, qui en dehors du contexte des inventaires sacrés, ne s'applique pas seulement aux divinités mais à la préparation de toutes les femmes qui se mettent en ordre, en arrangeant leur corps, leur visage, leurs cheveux, selon des pratiques codifiées. Le résultat est une apparence conforme à l'ordre voulu et acceptée par tous. C'est un terme relativement rare dans cette acception ${ }^{17}$.

Dans les inscriptions de Délos, il s'agit donc plus d'une préparation rituelle de la statue de marbre que de son ornementation et le mot ne s'applique qu'à Artémis et Héra ; dans un compte de l'Artémision: " pour la kosmesis de la statue, éponges 2 ; conge et demi

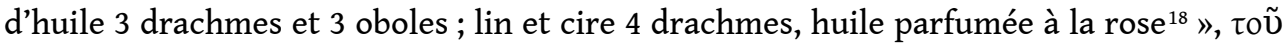

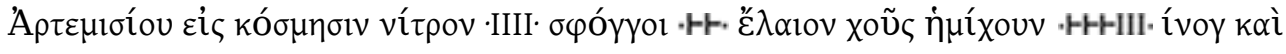

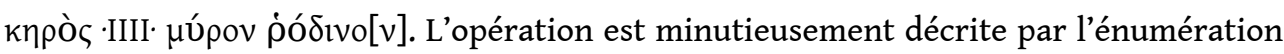

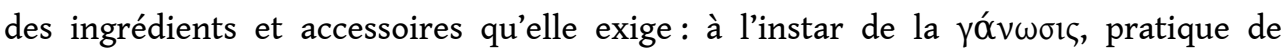

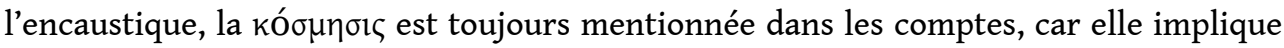

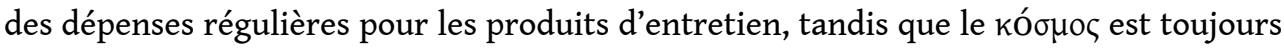
enregistré parmi les offrandes. 


\section{Kosmema, « ornement », « bijou »} tardif dans les sources littéraires, souvent métaphorique ${ }^{19}$, et Platon est un des premiers auteurs à l'employer en évoquant des ornements militaires ${ }^{20}$. Étonnamment, il est rarissime dans les sources épigraphiques et les inventaires sacrés ne l'utilisent jamais : une dédicace de Scythie mineure évoque peut-être un kosmema offert à Létô par Sôthemis, épouse de Protogenès mais la pierre comporte une lacune fâcheuse qui ne permet de lire que kó $\omega \mu[\eta \mu \alpha]^{21}$.

\section{La parure}

Les descriptions de bijoux dans les inventaires déliens et attiques sont caractérisées par la récurrence de certains éléments terminologiques qui permettent notamment aux administrateurs de les distinguer d'une année à l'autre lors du passage en revue des offrandes de tous les temples et d'ainsi prouver leur probité. De façon systématique, on mentionne le type de bijou, la matière et le poids; de façon fréquente, on y ajoute le nom du donateur (ou de la donatrice), le mode de présentation du bijou et son état lors de l'examen annuel.

Les multiples combinaisons de ces critères constituent ainsi un faisceau d'indices en faveur soit d'une fonction strictement votive (et donc d'un bijou destiné à la statue de culte), soit d'une sorte de remploi après usage privé, en marquant ainsi le lien affectif qui lie l'objet dédié à son donateur. En effet, il n'existe pas d'artisanat religieux systématique pour les bijoux, à l'instar des figurines qu'on achetait pour les dédier ; bien souvent, les bijoux du quotidien comme les fibules sont déposés en acte gratulatoire par les donateurs "ordinaires", c'est-à-dire évoluant dans la sphère privée, tandis que les bijoux fabriqués sur commande sont davantage des offrandes de prestige relevant de personnages célèbres voulant faire montre de leur piété envers une divinité: la description du vécu d'une offrande peut aider à comprendre sa destination finale et grâce à quelques combinaisons de critères présentées ici, on tentera de montrer comment la syntaxe des inventaires déliens pour les éléments de parure peut servir les rédacteurs de ces textes, et au-delà d'eux-mêmes, peut contribuer à comprendre la fonction des bijoux dédiés.

\section{Type}

Dans les inventaires déliens, de façon schématique, on peut distinguer deux grandes catégories de bijoux : ceux qui peuvent être portés au quotidien ${ }^{22}$ (bijoux ordinaires) et ceux qui se démarquent par leurs qualités stylistiques, l'originalité du motif, la prouesse de l'artisan ou encore, par exemple, les circonstances de la dédicace (bijoux singuliers).

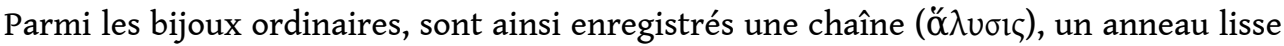

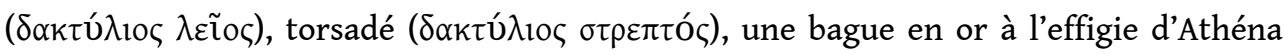

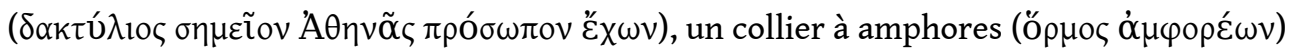

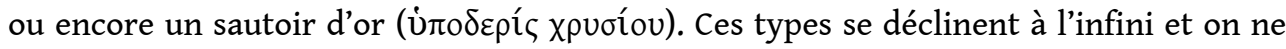
relève pas moins de 26 variantes d'anneaux catalogués ${ }^{23}$. Dans la majorité des cas, le

Cahiers « Mondes anciens », 15 | 2022 
nom du donateur n'est pas mentionné et nous ne disposons pas d'informations supplémentaires sur le don.

Dans la catégorie des bijoux singuliers, deux exemplaires retiennent l'attention par leur fonction, strictement votive dans le premier cas car destiné au kosmos d'Artémis, et propitiatoire dans le second car destiné à attirer la bienveillance de Létô ${ }^{24}$.

Dans l'Artémision, sur près de 40 années ${ }^{25}$, est enregistré un « collier en or suspendu au

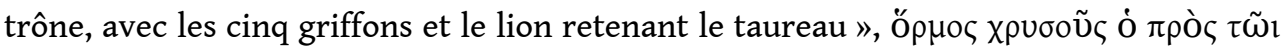

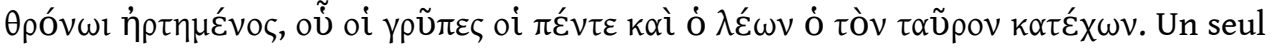
inventaire précise que les figures animales font partie d'un kosmos, en évoquant ainsi un ensemble de parure composite rassemblé autour de la statue de la déesse ${ }^{26}$. Les éléments qu'il comporte suggèrent qu'il ne s'agit pas d'un bijou typiquement hellénistique mais d'un collier d'inspiration plus ancienne et ionienne : les motifs du griffon et du lion sont propres aux styles insulaires à influence orientale. Nous avons sans doute affaire à une imitation de parure de type archaïque reproduite à l'époque hellénistique, comme cela se faisait également pour les sculptures.

La mention de son lieu d'exposition sur le trône de la déesse ${ }^{27}$ et son caractère stylistique particulièrement original sont des indicateurs en faveur d'une fonction strictement votive du bijou qui n'a pas été porté : il est destiné à orner la statue de culte qui trônait sur un siège et a vraisemblablement été fabriqué pour l'occasion.

Dans un inventaire tardif de l'Artémision ${ }^{28}$, les administrateurs athéniens cataloguent un «collier serti non pesé, avec une bande [de maille] qui y est attachée, 48 disques, deux moitiés et au centre, un seul disque et des pendeloques ornementales, dont le poids [est calculé] avec les trois myrtes tombés qui sont aussi dans le protège-anches à

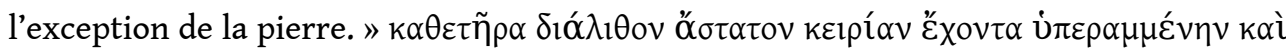

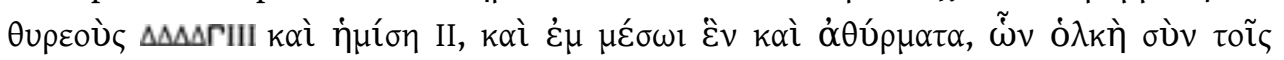

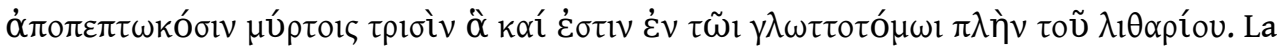
description minutieuse correspond au goût de l'époque de la seconde domination athénienne (314-166 av. J.-C.) où les rédacteurs enregistrent les dons à grand renfort de détails, pour ainsi souligner que l'île sacrée demeure un sanctuaire de prestige même quand elle n'est plus gérée par les Déliens. C'est en revanche grâce aux textes de la période de l'Indépendance délienne qu'on apprend l'origine de ce collier complexe: dédié à Létô par Stratoniké, épouse d'Antiochos $\mathrm{I}^{\mathrm{er}}$, il serait ainsi un cadeau propitiatoire offert à l'occasion du mariage de sa fille Stratoniké II avec Démétrios II en 253 av. J.-C.

\section{Matière}

L'examen des variations lexicales est mené sur un seul type d'objet offert pour montrer combien sont délicates les interprétations de l'usage des bijoux dans les inventaires.

L'épingle, désignée par le mot $\pi \varepsilon \rho o ́ v \eta^{29}$ constitue une des offrandes les plus fréquentes en raison des multiples lectures possibles qu'elle présente : selon sa matière, sa facture, le nombre dédié, sa provenance (privée ou commerciale) et sa destination, elle allie richesse, apparat et symbolique, en liant le dédicant à la divinité dans un geste métaphorique.

Dans l'Artémision, entre un fragment de motif floral et le collier d'Ériphyle, sont

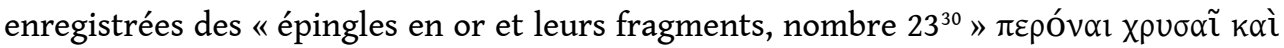




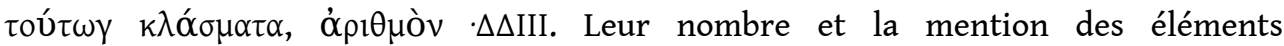
fragmentaires laissent présumer qu'elles n'ont pas été fabriquées pour l'occasion : il ne s'agit pas d'un lot votif mais de plusieurs bijoux rassemblés après dédicace pour la commodité du rangement dans le temple et pour l'inventoriage ensuite. Il est probable que ces fibules ont été dédiées individuellement, voire portées précédemment : si l'on en croit le degré d'usure de certaines dont il ne subsiste plus que les morceaux, $\kappa \lambda \alpha ́$ ó $\mu \alpha \tau \alpha$, elles ont subi maintes manipulations avant d'être offertes.

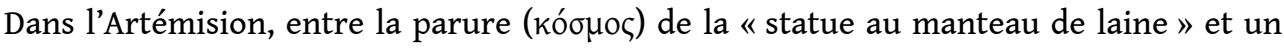
anneau en or, on relève une "épingle en argent doré, poids $15 \mathrm{dr} .^{31}$ ", $\pi \varepsilon \rho o ́ v \eta ~ \dot{\alpha} \rho \gamma v \rho \tilde{\alpha}$

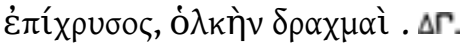

Le flou terminologique fréquent dans les inventaires déliens, fait que le même objet ressurgit dans un autre inventaire postérieur de 6 ans, rangé dorénavant entre le " collier aux lions et au taureau» (parure de la statue) et deux colliers : «fibule en

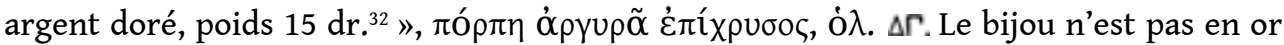
pur mais dans un placage fréquent parmi les offrandes dédiées ${ }^{33}$. Sa matière n'est cependant pas un indice en faveur d'une destination ou d'une autre (votive ou pour parer la statue) mais c'est uniquement sa place dans les inventaires - à la suite d'abord du kosmos de la statue au vêtement de laine puis à côté d'un collier dont on sait qu'il pare la statue - qui signale clairement qu'il fait partie de cet ensemble d'apparat ${ }^{34}$.

Dans l'oikos des Andriens, avant des ancres en fer, sont cataloguées des «épingles en

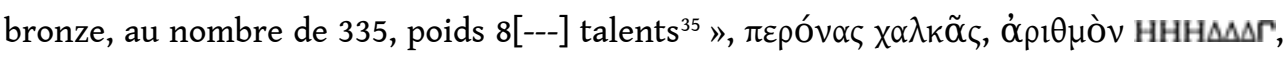

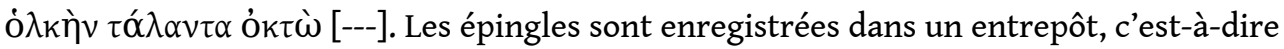
un lieu de stockage sans fonction religieuse: cela n'induit toutefois aucune désacralisation des offrandes qu'on y dépose régulièrement faute de place dans les temples. La matière des épingles est la plus commune, utilisée pour les offrandes issues d'un artisanat de masse et destinées à un usage quotidien. Le contexte de rangement (avant des ancres) signifie également qu'il n'y a pas de fonction ornementale pour la statue : par commodité, les hiéropes ont regroupé en cet endroit plusieurs séries qui ne proviennent pas des mêmes lieux.

Hors du champ d'études délien, les autres catalogues confirment que la matière peut difficilement constituer un indice fiable pour déterminer la destination d'un bijou. Dans un inventaire du $\mathrm{v}^{\mathrm{e}} \mathrm{s}$. av. J.-C. des temples de Mnia et Auzesia à Égine ${ }^{36}$, de nombreuses lignes sont réservées à la description des épingles aux usages multiples.

Dans l'opisthodomos : «épingles en fer, cent-vingt ; à côté des peploi : épingles en fer,

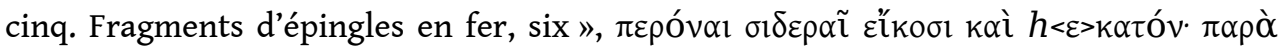

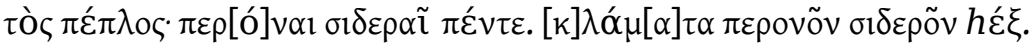

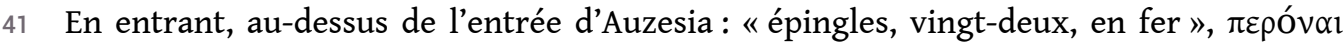

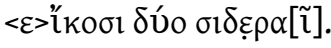

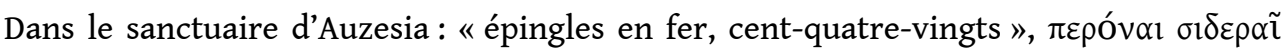

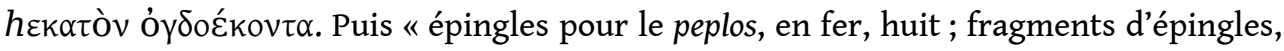

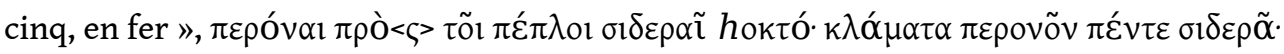

Il y a donc en tout 335 épingles intactes et 11 fragments enregistrés dans cet inventaire. La question de la destination de ces bijoux a longtemps fait débat : l'idée proposée par Paul Jacobsthal (1956, p. 98-99) ${ }^{37}$ d'un ensemble de vêtements accrochés aux murs et d'épingles déposées auprès d'eux n'est pas invraisemblable pour justifier la formule « à 
côté des peploi» puisque maintes offrandes étaient suspendues sur les murs des temples ${ }^{38}$; mais vêtements et épingles peuvent également être rangés sur une même étagère et être recensés normalement lors de l'exetasmos kata topon: un inventaire attique nous confirme d'ailleurs l'existence d'une "peplothèque ${ }^{39}$ ». L'hypothèse lancée par Irene Polinskaya (2013) d'épingles votives destinées aux deux divinités n'est pas incompatible avec la première proposition: cela expliquerait le regroupement par grands lots de spécimens $(120,180)$. Entre les deux, le nombre des épingles constitue néanmoins un bon indice pour l'interprétation de leur fonction; si la formule " épingles pour le peplos, en fer, huit » fait immédiatement songer à des offrandes par destination et donc affectées au kosmos de la statue de culte, les cinq autres épingles enregistrées "à côté des peploi » posent la question de la nature des vêtements mentionnés : plutôt que de songer à de simples peploi déposés en offrande, j'aurais tendance à les considérer comme faisant également partie du kosmos des statues qui comportait plusieurs tenues pour la divinitét ${ }^{40}$. Le matériau de ces épingles appelle un commentaire. Selon Paul Jacobsthal (1956), le fer implique un caractère ancien pour ces offrandes qu'il imagine être alors un lot transféré d'un sanctuaire proto-géométrique. Pour Cecilie Brøns (2016), il pourrait s'agir d'éléments strictement votifs conçus dans un matériau archaïque sans qu'elle n'en explique la raison.

De fait, le fer est souvent considéré comme un métal qui n'a plus cours à partir de l'époque classique : chez Homère, il revêt un caractère aussi précieux que l'or mais est abandonné ensuite au profit du bronze. Néanmoins, les inventaires de temples tendent à nuancer cette répartition chronologique en enregistrant fréquemment des bijoux de fer. À Délos, du IV $\mathrm{au} \mathrm{II}^{\mathrm{e}} \mathrm{s}$. av. J.-C., bon nombre de bagues sont en fer, parfois recouvert d'or ou d'argent mais parfois apparent ${ }^{41}$, et il est utile aussi de rappeler la valeur hautement symbolique de la bague en fer chez les Romains ${ }^{42}$.

En remettant ainsi en perspective le fer des épingles des divinités éginètes, l'usage de ce matériau dans un contexte classique est ambigu : considéré comme un métal de prix, il se justifie dans une dédicace destinée à faire partie du kosmos de la statue ; considéré comme un métal ancien de faible coût, il entre dans la composition d'un bijou issu d'un artisanat de masse, au même titre qu'une phiale ou un anneau de bronze qui seront déposés dans le temple.

Cependant, le caractère précieux d'un matériau ne prouve pas toujours qu'un bijou soit destiné à parer la statue de culte. En témoigne le caractère factice de certains éléments de parure des statues qui n'ont pas été portés et sont donc fabriqués dans un matériau de peu de valeur : c'est le cas du placage, qu'il soit d'or sur fer ou sur bronze, d'argent sur bronze, etc. mais aussi du bois doré, réservé aux bijoux portés par les statues de culte. Ainsi, dans le Thesmophorion de Délos, les statues de Déméter et Kore portent des pendants d'oreilles en bois doré : «statues des deux déesses sur leurs trônes, avec en acrolithe des couronnes de bois doré et des pendants d'oreille en bois doré, drapées

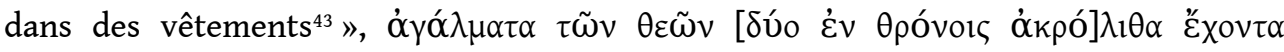

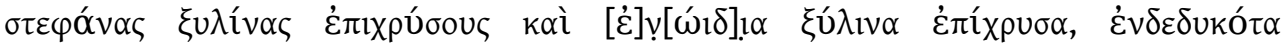
$\dot{\varepsilon} v \delta \dot{\mu} \mu \alpha \tau[\alpha]$. De la même façon, dans le sanctuaire d'Agathè Tychè, la statue de la déesse

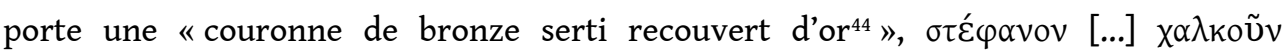

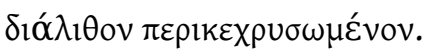




\section{Poids et nombre}

47 En continuant de croiser les multiples éléments récurrents décrivant les bijoux dans les inventaires déliens, il s'agit de voir comment, après le type ou le matériau, le poids ou le nombre, combinés aux précédents, peuvent être des signes plus solides de la fonction d'une parure déposée dans un temple.

Si nous reprenons l'exemple du collier aux griffons précédemment exposé, outre l'indication spatiale "suspendu au trône» et la riche facture de la parure, il faut admettre que l'offrande sort de l'ordinaire en raison de son poids également, qui est de 210 drachmes en moyenne ${ }^{45}$, équivalant à 930 grammes environ. Un bijou de ce poids dans un contexte votif ne se conçoit pas comme une pièce qui aurait pu être portée. Cela confirme qu'il ne constitue pas une offrande relevant de la sphère privée et dédiée personnellement par un individu exprimant sa reconnaissance, mais bien un don pour la déesse elle-même et qui fait partie de son kosmos.

Une offrande de la reine Stratoniké, composée de « vingt médaillons en onyx enchâssés dans de l'or, auxquels est attachée une chaîne en or, poids avec les anneaux $430 \mathrm{dr} .2$

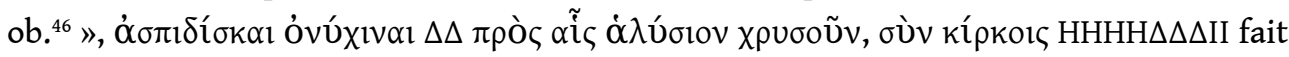
émerger la combinaison de deux autres critères : le poids, dans la mesure où le collier pèse près de $2 \mathrm{~kg}$, et l'identité de la donatrice, personnage célèbre qui vient contribuer au prestige de l'île par l'offrande d'une riche parure strictement votive.

À côté des indications de poids qui vont en faveur de dons par destination lorsqu'ils sont lourds, le nombre de bijoux enregistrés permet également de déterminer s'ils sont prévus pour la statue de culte ou s'il s'agit d'offrandes banales et de masse.

Dans le temple des Athéniens, après l'énumération des couronnes des vainqueurs des jeux, on enregistre «diadèmes et couronne, 103, qu'a offerts Nicias, diadèmes qu'a

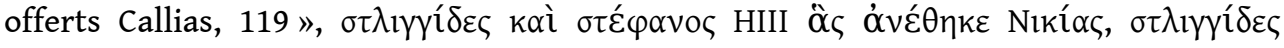

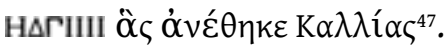

Il s'agit d'un don de Nicias et Kallias, les deux archithéores venus à Délos avec le chœur de musique de la cité d'Athènes pour la représenter lors des concours d'hymnes en l'honneur d'Apollon ; être théore était certes une distinction pour un citoyen ordinaire, mais Nicias et Callias ont fait preuve envers le sanctuaire délien d'Apollon de largesses très inhabituelles pour le $\mathrm{V}^{\mathrm{e}}$ siècle comme le rapporte Plutarque (Vitae, Nicias IV). Le cas est intéressant pour la compréhension de la fonction de ces couronnes car il ne s'agit ni d'un don individuel et personnel - Nicias et Callias n'ayant évidemment pas porté tous ces diadèmes et couronnes -, ni de la récompense aux concours musicaux : bien qu'ils aient mené les chœurs athéniens dans les concours de Délos, le nombre de couronnes et diadèmes est trop important pour constituer le prix de leur victoire. Nous sommes là en présence d'un don par destination mais qui n'appartient pas au kosmos de la statue de culte d'Apollon, dans une sorte de degré ultime de la dédicace.

On retrouve le même don au $\mathrm{II}^{\mathrm{e}}$ siècle av. J.-C. dans le temple aux Sept Statues, avec un mode de présentation décrit, qui confirme l'intention votive stricte des couronnes : bon nombre d'offrandes sont ainsi conçues à Délos, comme les boucliers offerts à la suite d'une victoire par exemple, mais il est rare d'observer ce phénomène dans la catégorie des parures, généralement destinées à la statue de culte ou représentant un don de bijoux personnels: «le plus grand nombre de diadèmes de théorie sur un panneau, 
offrande de Kallias l'Athénien, fils d'Hipponikos» (Coupry 1954, p. 290), $\sigma \tau \lambda \varepsilon \gamma \gamma \hat{\delta} \delta 1 \alpha$

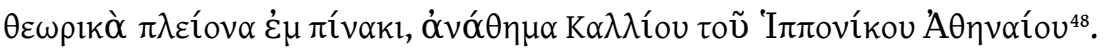

\begin{tabular}{|l|l|l|}
\hline BIJOU & Simple offrande d'après les inventaires & Fabrication pour la statue de culte \\
\hline Type & Ordinaire : - déjà porté - stéréotypé & Singulier (facture, style, etc.) \\
\hline Matière & Métal massif (or, argent). Placage & $\begin{array}{l}\text { Placage sur métal ou bois. Métal massif } \\
\text { (bronze, or) }\end{array}$ \\
\hline $\begin{array}{l}\text { Poids/ } \\
\text { nombre }\end{array}$ & $\begin{array}{l}\text { Cohérent avec un usage. Grand nombre } \\
(\text { lot })\end{array}$ & Lourd / unicum \\
\hline Donateur & Donateur (+ ethnique) & $\begin{array}{l}\text { Donateur (+ patronyme) + divinité } \\
\text { bénéficiaire }\end{array}$ \\
\hline Présentation & $\begin{array}{l}\text { Libre vs fixé sur présentoir (bandelette, } \\
\text { planche, tissu...) }\end{array}$ & $\begin{array}{l}\text { Libre, indication spatiale (près du trône, } \\
\text { dans la main, etc.) }\end{array}$ \\
\hline État & Usé, détaché, éléments manquants & Intact ou mis dans une boîte \\
\hline
\end{tabular}

En conclusion, tentons de résumer grâce au tableau ci-dessus les multiples critères permettant d'affiner la compréhension du rôle des parures dans les inventaires déliens : la nature donne la fonction, pourrait-on schématiser. Il faut toutefois garder à l'esprit que les frontières entre les usages des bijoux dans les temples sont parfois minces et que les catégories et correspondances proposées ici de façon schématique ne sont ni figées ni systématiques : seule l'étude croisée des différentes informations sur les bijoux permet d'imaginer de façon un peu plus précise les intentions des administrateurs du sanctuaire lorsqu'ils enregistrent des parures. Qu'il s'agisse de la description d'un bijou porté auparavant par le dédicant, qu'il soit destiné au kosmos de la statue de culte, qu'il résulte d'un artisanat en série à l'instar des figurines de terre cuite, l'analyse combinée des matières, types ou encore mode de présentation dans le temple démontre une fois encore l'ingéniosité lexicale des rédacteurs des inventaires, en dépit des contraintes imposées par le genre.

\section{Abréviations}

ID : Corpus des Inscriptions de Délos, Paris (1926-1937).

IG : Inscriptiones Graecae, vol. XI (Délos), Berlin (1912-1914). 


\section{Bibliographie générale}

Avram A. (2003), « An Istrian Dedication to Leto », dans Bilde P. G., Højte J. M. et Stolba V. F. éd., The Cauldron of Ariantas, Studies Presented to A.N. Sceglov on the Occasion of his 70th Birthday, Black Sea Studies 1, Aarhus, p. 87-91.

Bodiou L., Gherchanoc F., Huet V. et Mehl V. (2011), Parures et artifices : le corps exposé dans l'Antiquité, Paris.

Brøns C. (2016), Gods and Garments. Textiles in Greek Sanctuaries in the 7th to the 1st Centuries BC., Ancient Textiles Series 28, Oxford.

Bruneau P. (1970), Recherches sur les cultes de Délos à l'époque hellénistique et à l'époque impériale, BEFAR 217, Paris.

Casevitz M. (1989), «À la recherche du kosmos. Là tout n'est qu'ordre et beauté », Le temps de la réflexion $\mathrm{X}$, Paris, p. 97-119.

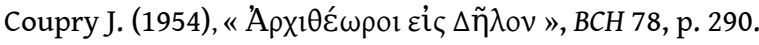

Deloche M. (1896), Le port des anneaux dans l'Antiquité romaine et dans les premiers siècles du Moyen Âge, Mémoires de l'Institut de France 35-2, Paris.

Donohue A. A. (1988), Xoana and the Origins of Greek Sculpture, American Classical Studies 15, Atlanta.

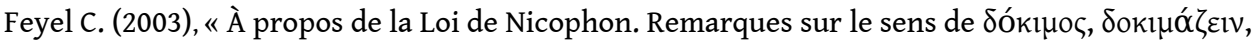

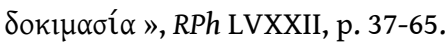

Grubbs J. E. et Parkin T. éd. (2013), The Oxford Handbook of Childhood and Education in the Classical World, Oxford.

Grand-Clément A. (2011), La fabrique des couleurs. Histoire du paysage sensible des Grecs anciens (VIIIedébut du Ve siècle av. n. è.). De l'archéologie à l'histoire, Paris.

Jacobsthal P. (1956), Greek Pins and their Connexions with Europa and Asia, Oxford.

Kron U. (1996), « Priesthoods, Dedications and Euergetism: What Part Did Religion Play in the Political and Social Status of Greek Women? », dans Hellström P., Alroth B. éd, Religion and Power, Uppsala, p. 139-182.

Morel J.-P. (1992), « Ex-voto par transformation, ex-voto par destination (à propos du dépôt votif de Fondo Ruozzo à Teano), dans Mactoux M.-M et Geny E. éd., Mélanges Pierre Lévêque, Besançon, p. 221-232.

Mylonopoulos I. (2009), «Introduction », dans Mylonopoulos I. éd., Divine Images and Human Imaginations in Ancient Greece and Rome, Religions in the Graeco-Roman World 170, Leyde, p. 1-20.

Polignac F. de (2009), «Quelques réflexions sur les échanges symboliques autour de l'offrande », dans Prêtre C. éd. (2009), Le donateur, l'offrande et la déesse, Kernos Suppl. 23, Liège, p. 29-37.

Polinskaya I. (2013), A Local History of Greek Polytheism: Gods, People and the Land of Aigina, 800-400 $B C E$, Religions in the Graeco-Roman World 178, Leyde.

Patera I. (2012), Offrir en Grèce ancienne. Gestes et Contextes, Stuttgart.

Prêtre C. éd. (2009), Le donateur, l'offrande et la déesse, Kernos Suppl. 23, Liège.

Prêtre C. (2012), Kosmos et kosmema. Les offrandes de parure dans les inventaires déliens, Kernos Suppl. 27, Liège. 
Prêtre C. (2014), «L'offrande dans les inventaires de Délos : objet rituel ou objet sacré ? », RHR 4, p. 539-557.

Satlow M. L. éd. (2013), The Gift in Antiquity, Oxford.

Simon C. G. (1986), The Archaic Votive Offerings and Cults of Ionia, Ph.D. diss., University of California, Berkeley.

Tréheux J. (1956), « L'inventaire des Clérouques d'Imbros », BCH 80, p. 462-479.

Tréheux J. (1991), « Retour à Imbros et à Samothrace ", dans Goukowsky P., Brixhe C. éd, Hellènika Symmikta. Histoire, archéologie, épigraphie, Nancy, p. 143-149.

\section{NOTES}

1. Pour la remise en perspective des théories maussiennes et de leurs apports pour l'étude de l'Antiquité, voir par exemple Satlow 2013.

2. Simon 1986, p. 199 et 221 ; Kron 1996, p. 159.

3. L'inverse est également vrai : dans les Sept contre Thèbes, Étéocle fait la dédicace des armes et armures ennemies dans les sanctuaires de la cité et dans une épigramme de l'Anthologie, Arès s'étonne de voir déposer en son honneur « des gâteaux délicats, une grappe de raisin et des couronnes de roses $"$.

4. Pour une définition du terme, voir Patera 2012, p. 19.

5. La distinction entre les deux dans un contexte épigraphique n'est pas aisée à faire : dans la majorité des cas, on ignore si l'offrande a été faite en amont afin de s'attirer les faveurs de la divinité (propitiatoire) ou s'il s'agit d'un ex-voto une fois un souhait exaucé (gratulatoire).

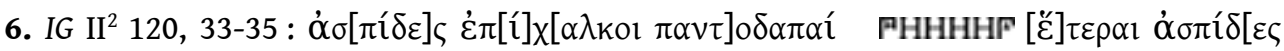

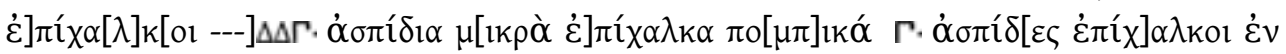

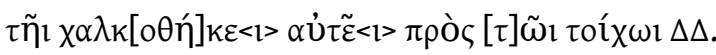

7. Sens délien plus précis.

8. À l'origine, collier d'Harmonie, fille d'Arès et d'Aphrodite, reçu pour ses noces avec Kadmos, puis donné ensuite en cadeau à Ériphyle pour qu'elle force son époux Amphiaraos à participer à la guerre des Sept contre Thèbes. Il s'agit donc bien d'un collier destiné à une divinité : sa qualification de kosmos au sein des inventaires déliens est donc justifiée.

9. Bijou complexe et d'interprétation délicate. Cf. Prêtre 2012, s.v.

10. IG XI2, 161B (62) et 162B (50).

11. Bruneau 1970, p. 176. Pour le xoanon, cf. Donohue 1988.

12. ID 367 (28); 410bis (4); 439c (4); 442B (200); 443Bb (124); 444B (43); 461Bb (31).

13. IG II ${ }^{2}, 1213(1)$.

14. IG $\mathrm{II}^{2}, 1328$ (11).

15. Ayepuó désigne ici l'ensemble des biens que possède une divinité, et notamment les monnaies et les bijoux. 
16. Cf. par exemple une œnochoè à figures rouges du Peintre de Mannheim, où Polynice soudoie Ériphyle avec le collier; Paris, Musée du Louvre, inv. G 442, https:// collections.louvre.fr/ark:/53355/cl010270291.

17. Cf. Grand-Clément 2011, p. 277-278, pour un commentaire détaillé de l'action à Délos.

18. IG XI 2, 161A (92).

19. Lucien, De la danse, 32 , au sens de gloire, lustre.

20. Platon, Lois, XII, 956b : «On n'emploiera d'autres teintures que pour les ornements militaires. "

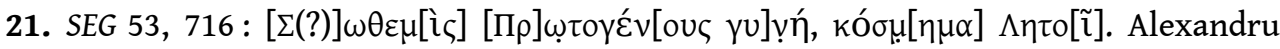
Avram (2003, p. 87-91), considère même qu'il ne lit que ko̧ sur la pierre.

22. Ainsi que les lots de bijoux communs comme les fibules.

23. Passim dans les inventaires déliens entre 314 et 116 av. J.-C. on peut relever les

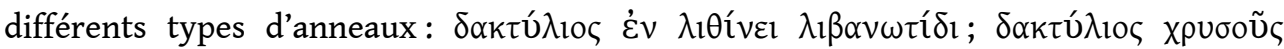

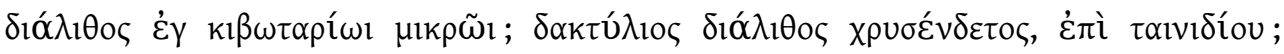

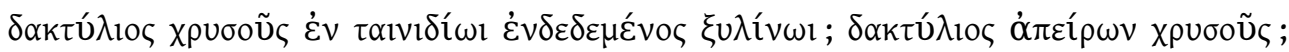

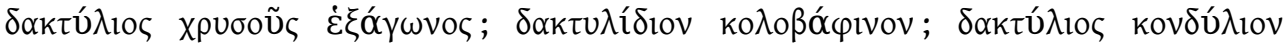

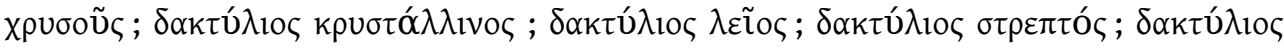

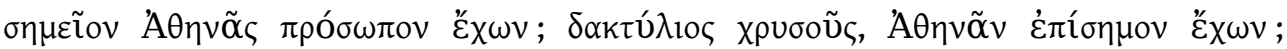

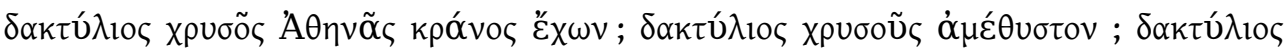

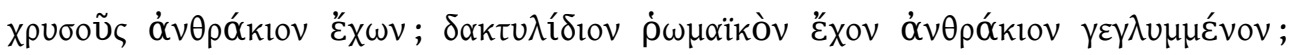

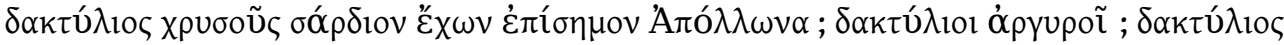

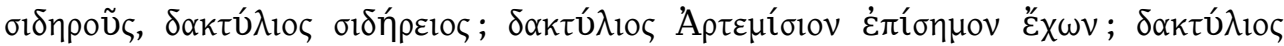

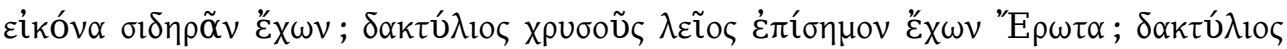

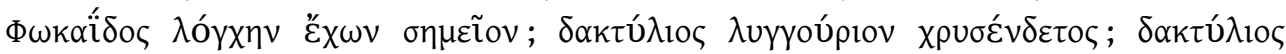

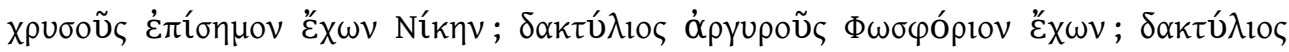

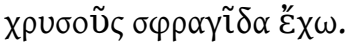

24. Les offrandes peuvent en effet avoir une fonction propitiatoire lorsqu'elles sont offertes dans le but de faire exaucer un souhait ou gratulatoire lorsqu'elles agissent comme ex-voto.

25. Première occurrence en 281 av. J.-C., IG XI 2,161B (22). Dernière occurrence en 143 av. J.-C., ID 1443B(I) (105). Entre les deux, quelques variations pour l'enregistrement du poids mais la description reste à peu près stable.

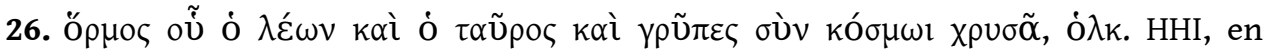
ID 399Ba (135).

27. Felix Durrbach glose l'offrande lors de sa première mention; IG XI 2,161B (22): torquis nempe sellae appensus in qua simulacrum deae sedebat.

28. ID 1432Aa (20), mais le collier existait avant, dès l'inventaire ID 298A (145), avec une description plus réduite.

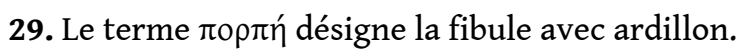

30. IG XI2, 161B (41).

31. IG XI2, 161B (62); 162B (50).

32. ID 407 (8). 
33. Il s'agit d'une technique de dorure à la feuille appliquée sur divers matériaux : ivoire, bois, bronze, argent, même si dans ce dernier cas, la matière n'est pas toujours mentionnée tant elle est évidente.

34. Son poids est approximativement de 65 grammes : cela ne plaide pas en faveur d'un usage quotidien.

35. IG XI2, 298A (179).

36. IG I ${ }^{3}$ 1455. Pour un commentaire de ce texte, cf. Polinskaya 2013, p. 615-622.

37. On ne retiendra pas l'argumentum a silentio de Cecilie Brøns (2016, p. 421) : «It seems that there are only few peploi in relation to the dress-fasteners recorded in the inventory, and the two clearly do not correspond in number.»; on ne cessera de répéter contre ce genre de sophisme que les inventaires n'étaient pas exhaustifs mais constituaient un choix d'offrandes. Le fait que les peploi n'aient pas été enregistrés sur la pierre ne signifie en rien qu'ils n'existaient pas dans le temple.

38. Miroir en IG II $^{2} 1515$ (16), ex-voto anatomiques en IG II $^{2} 1534$ A (49), boucliers en IG II $^{2} 1469$ (68).

39. IG II 1462 (12). Pour le fonctionnement de l'exetasmos, voir en premier lieu Tréheux 1956, p. 462-479 et Tréheux 1991, p. 143-149, ainsi que les commentaires de Feyel 2003, p. 37-65 et notamment p. 40.

40. Un décret attique en l'honneur des Parthenoi qui tissent le peplos d'Athena évoque le

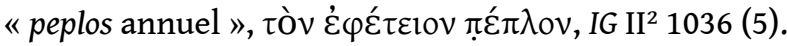

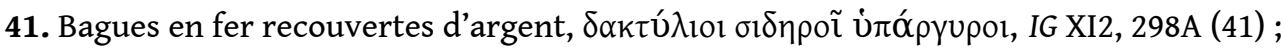

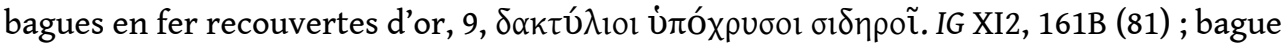

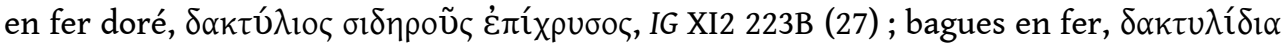

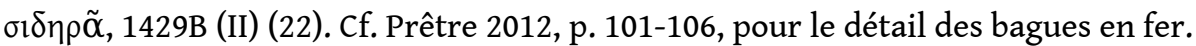

42. Cf. la monographie vieillie mais néanmoins pertinente de Maximin Deloche (1896, p. 172 notamment).

43. ID 1424B (4).

44. ID 1403B (II) (11).

45. Les variations de poids sont fréquentes dans les inventaires et celui-là oscille entre 201 et 214 drachmes.

46. IG XI2, 287B (70).

47. ID 101 (39).

48. ID 1409Ba (II) (57).

\section{RÉSUMÉS}

À travers l'examen des descriptions de bijoux dans les inventaires d'offrandes de Délos à l'époque hellénistique, il s'agit d'identifier quels critères permettent de comprendre la fonction des parures déposées dans les différents temples de l'île : type, matière, mode de présentation, poids, état décrit, constituent autant d'indices qui, combinés, peuvent nous aider à déterminer si les 
bijoux sont des offrandes fabriquées à des fins strictement votives (pour orner les statues de culte), ou des offrandes extraites du quotidien de la sphère privée pour être dédiées dans un geste votif ordinaire.

Through the analysis of the jewels described in the sacred inventories of Delos dating back to the Hellenistic period, we will attempt to understand what criteria are instrumental in determining the function of the adornments recorded in different temples of the island: their type, material, mode of exhibition, weight and quality are all indices which, if combined, can help us to establish whether the jewels are offerings made for strictly votive purposes (to adorn the cult statues), or offerings coming from the daily private sphere to be dedicated in an ordinary votive gesture.

INDEX

Keywords : sacred inventory, epigraphy, greek religion, jewel, Delos

Mots-clés : inventaire sacré, épigraphie, religion grecque, bijou, Délos

\section{AUTEUR}

\section{CLARISSE PRÊTRE}

CNRS, ANHIMA UMR 8210 